\title{
Discriminating Among Single Locus Models Using Small Pedigrees
}

\author{
T. H. Beaty \\ Department of Human Genetics, University of Michigan, Ann Arbor
}

\begin{abstract}
Simulated small pedigrees ( 2 parents, 4 offspring) were used to illustrate the applications and limitations of a "model choice" approach designed to detect genetic heterogeneity in familial diseases. While it is possible to identify groups of pedigrees which have different genetic causes for quantitative phenotypic trait(s), theoretical limitations on discriminating between 4 single locus models exist for certain pedigree structures. These limitations originate from the overlapping phenotypic predictions of the various genetic models. Such limitations must be carefully considered in the design of genetic studies. Studies aimed at detecting genetic heterogeneity in familial diseases should limit the different genetic models being considered and tailor the sampling strategy to avoid collecting pedigrees which are non-informative for certain comparisons.
\end{abstract}

Key words: likelihood analysis, pedigree analysis, multivariate phenotypes, genetic heterogeneity

\section{INTRODUCTION}

A major goal in pedigree analysis today is to identify genetic mechanisms which may underlie the familial aggregation seen in several of the common diseases such as diabetes mellitus. While such familial aggregation may be striking when pedigrees denoting affected and unaffected individuals are examined, the findings of many genetic studies on such diseases have been inconsistent to date. Some of this inconsistency may be due to the loss of information accompanying the inevitably arbitrary definition of "affected" vs "unaffected" phenotypes based on an underlying quantitative phenotype such as blood glucose level. If such a quantitative phenotype is available, it will generally carry far more information about genotypic expression than will a qualitative diagnosis. Another, less tractable, explanation for this inconsistency is that several distinct genetic forms of the disease may exist in the population. This paper gives a brief description of an analysis designed to detect such genetic heterogeneity in familial diseases.

Received for publication August 20, 1979; revision received March 19, 1980.

Dr. Beaty is now at the Dept. of Epidemiology, School of Hygiene and Public Health, Johns Hopkins Univ., Baltimore, MD 21205 . Address reprint requests to this address. 
In the past decade, a series of workers have developed algorithms for evaluating genetic models on pedigrees by computing the likelihood function of the model in question [Elston and Stewart, 1971; Lange and Elston, 1975; Cannings et al, 1978]. To compute these likelihood functions, it is necessary to identify various parameters which are defined by any genetic model. There are three main classes of parameters: 1) the transition parameters describing the transmission of alleles from parent to offspring; 2) the frequency parameters describing the genotypic frequencies in the population being sampled; and 3) the penetrance parameters which describe the phenotypic expression of different genotypes. The particular genetic model in question defines the values of these parameters, or at least their possible ranges. For example, a Mendelian genetic model fixes the values of the transition parameter exactly, but allows a wide range of possible genotypic frequencies, one set of which represents the Hardy-Weinberg equilibrium point. Similarly, a dominant genetic model demands the penetrance parameters of certain genotypes be identical, but the exact form of the penetrance functions will be very different for quantitative phenotypes compared to those for a qualitative phenotype. Once these parameters are specified, the observed information on phenotypes and the relationships between individuals in a pedigree can be combined in a sequential conditioning or "peeling" process [Cannings et al, 1978] to give the total likelihood function of any genetic model on the entire pedigree.

These likelihood functions can be used either to estimate the parameters of a single genetic model or to choose between two competing models. In cases where the competing models are a general "complete" model and a specific "reduced" model (representing a subset of the "complete" parameter space), the likelihood ratio criterion offers a convenient and well-recognized test statistic for large numbers of pedigrees [Elston et al, 1975]. Not all genetic models fit these requirements, however, since many simple genetic models do not represent hierarchical parameter spaces. For example, the autosomal single locus model and the X-linked single locus model cannot be directly compared using the likelihood ratio criterion due to the differences in the number of genotypes in the two models and how they vary between the sexes.

Even though the likelihood ratio criterion cannot be applied to the comparisons of several common genetic models, it is possible to use a similar ratio of two likelihoods to choose the better supported model without knowing the exact distribution of such a "model choice" test statistic. This approach can be used to search for genetic heterogeneity within a single disease by sorting pedigrees into groups which favor one genetic model over another. In this paper, I shall describe how such a "model choice" approach could be used on pedigrees with quantitative phenotypes, discuss the improvement possible with multivariate phenotypes, and identify intrinsic limitations in this approach. To illustrate this "model choice" approach, small pedigrees ( 2 parents, 4 offspring) were simulated using Monte Carlo techniques under four single locus models (autosomal dominant and recessive, X-linked dominant and recessive). Simulated data were chosen because of the difficulties in obtaining analytical solutions when working with likelihood functions of even small pedigrees such as these when the phenotypes are quantitative.

\section{The "Model Choice" Test Statistic}

When simply choosing between two genetic models, a simple ratio of likelihoods of the two models can serve to indicate which model is better supported by the observed data. To ensure that such a comparison of likelihoods computed over two possibiy different parameter spaces is analogous to a comparison of the probabilities under the two models, however, some type of prior information on other parameters of the genetic model - such 
as allele frequency - should be incorporated [Beaty, 1978]. This prior information can be easily obtained from independent estimates of the prevalence of the disease being studied. For example, assuming Hardy-Weinberg equilibrium in the general population, a prevalence of $1 \%$ would imply the frequency of the mutant allele $\left(\mathrm{p}_{\mathrm{AD}}\right)$ is 0.005 if the disease were solely determined by an autosomal dominant allele. This same prevalence, however, would imply $\mathrm{p}_{\mathrm{AR}}=0.1$ under the autosomal recessive model. This simple type of prior information enables the "model choice" ratio statistic to be written as:

$$
\Lambda=\log \frac{\mathrm{L}\left(\operatorname{Model} 1 \mid \mathrm{p}_{1}\right)}{\mathrm{L}\left(\operatorname{Model} 2 \mid \mathrm{p}_{2}\right)}
$$

where $p_{1}$ and $p_{2}$ are the allele frequencies implied by the observed population prevalence under models 1 and 2, respectively. While this "model choice" statistic can be used to identify the more likely of the two models, we can make no general statement about its distribution.

\section{The Simulated Data}

Four sets of nuclear families ( 2 parents, 4 offspring) were simulated using Monte Carlo techniques under four single locus, two allele models: the autosomal dominant and recessive models (termed $\mathrm{AD}$ and $\mathrm{AR}$, respectively) and the X-linked dominant and recessive models (termed XD and XR, respectively). Previous studies have used this pedigree structure for estimating parameters of genetic models and for testing a variety of genetic hypotheses [Go et al, 1978; MacLean et al, 1975]. In each set of pedigrees, the appropriate genetic model determined the inheritance of a hypothetical disease whose frequency in the overall population was $1 \%$. Each pedigree was ascertained through an "affected" parent randomly selected from this $1 \%$ sub-population, while the other parent was randomly drawn from the total population. The total data set consisted of 160 pedigrees simulated under each of the two autosomal models, and 144 pedigrees simulated under each of the two sex-linked models (plus an additional 32 simulated under the XR model, so the rare female probands would be adequately represented).

In each pedigree, once an individual's genotype had been assigned under one of the four models, the observable phenotype was assigned. The phenotype consisted of four traits, each randomly drawn from one of two normal distributions. Those genotypes defined by the model as being "affected" were assigned phenotypic values from the distribution centered at 1.5 , with variance 1.0 , while other genotypes were assigned phenotypes from a distribution with the same variance but centered at zero. For example, under the AR model, homozygotes for the mutant allele were assigned phenotypes by four independent draws from the "affected" distribution, ie, the $\mathrm{N}(1.5,1)$ while under the AD model both the mutant homozygotes and heterozygous carriers were assigned phenotypes from this distribution.

Ultimately the ability to discriminate between genetic models must depend on these phenotypic differences between genotypes. With only 1.5 standard deviations between the means of these two distributions, it is quite difficult to distinguish between "affected" and "unaffected" individuals on the basis of a single trait, as illustrated in Figure 1a. By considering several phenotypic values simultaneously, however, the degree of phenotypic ambiguity can be substantially decreased. The separation between two multivariate distributions (with means $\underline{\mu}_{1}$ and $\underline{\mu}_{2}$, and common covariance matrix $\Sigma$ ), as measured by the Mahalanobis distance $\bar{\Delta}^{2}=\left(\underline{\mu}_{1}-\underline{\mu}_{2}\right)^{\prime} \Sigma^{-1}\left(\underline{\mu}_{1}-\underline{\mu}_{2}\right)$, can be used to convert the phenotypic 

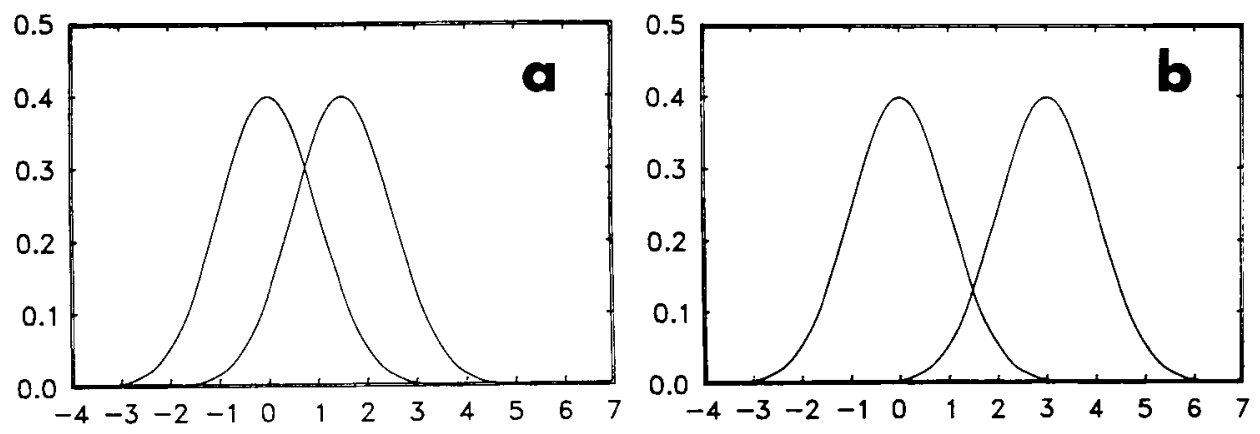

Fig. 1. a) Degree of overlap between two normal distributions with unit variance and 1.5 standard deviations between their means. b) Degree of overlap between two normal distributions with three standard deviations between their means. This latter situation has the same degree of separation which occurs in four-dimensional space between two quadrivariate distributions constructed by four independent draws from (a).

separation attained in multidimensional space back to a univariate scale. By letting $\Delta_{\mathbf{u}}$ represent the distance between the means of two univariate distributions, the relationship $\Delta_{\mathrm{u}} \sqrt{\mathrm{k}}=\Delta$ shows the separation attained by considering four independently drawn replicates (ie, $\mathrm{k}=4$ ) has doubled the phenotypic separation between the "affected" and "unaffected" phenotypes (see Fig. 1b). In general, a multivariate phenotype can either represent separate physical traits, if the assumption of joint control by a single genetic mechanism is tenable (eg, insulin and blood glucose), or it could be replicate measurements of a single trait, as was done here. By comparing the results of likelihood analysis done on these pedigrees with one phenotypic trait $(\Delta=1.5)$ to that using four traits simultaneously $(\Delta=$ 3.0 ), the effect of increasing phenotypic resolution can be examined for a fixed genotypic combination.

\section{RESULTS}

\section{Pairwise Comparisons of Genetic Models}

The frequency distribution of the "model choice" statistic comparing the AD and AR models $\Lambda=\log [\mathrm{L}(\mathrm{AD} \mid \mathrm{p}=0.005) / \mathrm{L}(\mathrm{AR} \mid \mathrm{p}=0.1)]$ for the 320 simulated autosomal pedigrees is shown in Figure 2. Here the univariate and quadrivariate phenotypes were used (Figs. 2a and 2b, respectively). When phenotypic resolution is poor, there is extensive overlap in the distributions of the statistic among the AD and AR pedigrees (Fig. 2a). When phenotypic resolution is improved, however, distinction between the $A D$ and $A R$ models becomes clearer, ie, AD pedigrees have more positive $\Lambda$ values and $\mathrm{AR}$ pedigrees have more negative $\Lambda$ values (where the magnitude of $\Lambda$ reflects the degree of support given to one model over the other). More important than the relative support given to a model, however, is the presence of absolute limits on $\Lambda$ which become obvious only when there is adequate phenotypic resolution (Fig. 2b).

These limits result from the ability of both models to explain the phenotypic combinations observed in the pedigree at slightly different frequencies. The exact values of these limits can be obtained from the likelihood functions themselves. Consider the phenotypic combinations possible in this pedigree under the AR model. For a rare disease, the most frequent phenotypic combination will be one "affected" parent (the proband), 

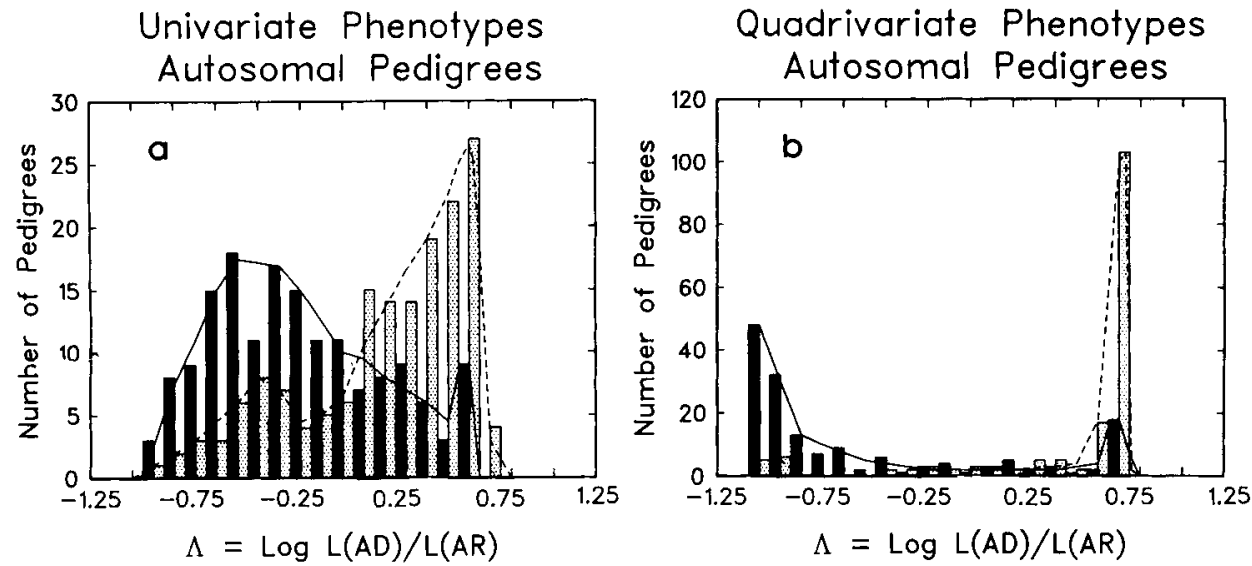

Fig. 2. Histogram of $\Lambda=\log L(A D) / L(A R)$ for 160 pedigrees simulated under the AD model (dotted bars) and 160 pedigrees simulated under the AR model (solid bars). a) Likelihoods computed using univariate phenotypes and $\mathrm{b}$ ) using quadrivariate phenotypes.

one "unaffected"parent, and four "unaffected" offspring (all heterozygotes). The frequency of this mating in a sample ascertained through an "affected" proband (homozygous for the mutant allele) is simply $q_{A R}^{2}$ (the frequency of the "unaffected" spouse). This same phenotypic combination can be explained by the AD model, however, in the mating of an "affected" heterozygote (the proband) and an "unaffected" homozygote, but at a much lower frequency because each child only has a 0.5 probability of not receiving the mutant allele under the AD model. The frequency of this particular phenotypic combination under the $\mathrm{AD}$ model is $\left\langle 2 \mathrm{p}_{\mathrm{AD}} \mathrm{q}_{\mathrm{AD}} /\left(\mathrm{p}_{\mathrm{AD}}^{2}+2 \mathrm{p}_{\mathrm{AD}} \mathrm{q}_{\mathrm{AD}}\right)\right\rangle \cdot\left\langle\mathrm{q}_{\mathrm{AD}}^{2}\right\rangle \cdot\left\langle(0.5)^{4}\right\rangle$, ie, (the frequency of a heterozygous proband) $\cdot$ (the frequency of a homozygous normal spouse) (the probability of 4 "unaffected" children). The "model choice" statistic $\Lambda[=\log \mathrm{L}(\mathrm{AD}) / \mathrm{L}(\mathrm{AR})]$ will be bounded from below by the $\log$ of the ratio of these two frequencies, regardless of the degree of phenotypic resolution.

In Figure $2 \mathrm{~b}$, many of the $160 \mathrm{AR}$ pedigrees are at this lower limit $(=-1.12$ for $\mathrm{p}_{\mathrm{AD}}=0.005$ and $\mathrm{p}_{\mathrm{AR}}=0.1$, where the AR model is 13 times more likely than the AD model). Whenever substantial ambiguity exists between the "affected" and "unaffected" phenotypes, this $\Lambda$ statistic may have some value above this limit, as in Figure 2a.

As also seen in Figure 2, there is a similar upper limit on the $\Lambda$ statistic in this comparison. This upper limit again results from the ability of both genetic models to explain the observed combinations of phenotypes. Among the AD pedigrees, the most frequent phenotypic combination would be: one "affected" parent, one "unaffected" parent, and an equal mixture of "affected" and "unaffected" offspring. This same combination can be predicted under the AR model if the "unaffected" parent is a heterozygote. Here, the "model choice" statistic will be bounded from above by the log of the ratio $\left\langle\left[2 \mathrm{p}_{\mathrm{AD}} \mathrm{q}_{\mathrm{AD}} /\right.\right.$ $\left.\left.\left(p_{A D}^{2}+2 p_{A D} q_{A D}\right)\right] \cdot q_{A D}^{2}\right\rangle /\left\langle 2 p_{A R} q_{A R}\right\rangle$, which is 0.739 for these data where $p_{A R}=0.1$ and $\mathrm{p}_{\mathrm{AD}}=0.005$. At high levels of phenotypic resolution, most of the $160 \mathrm{AD}$ pedigrees are at this upper limit. Furthermore, looking closely at Figure $2 b$ shows several among the 160 AR pedigrees which have a $\Lambda$ value at this upper limit, rather than near the lower limit described earlier. In these AR pedigrees the randomly chosen spouse of the proband was indeed a heterozygote, so these pedigrees do have an equal mixture of "affected" and 
"unaffected" offspring just like the AD pedigrees. Of the 160 mating types in the AR pedigrees, 28 were homozygote $X$ heterozygote and one was a mating between two individuals both homozygous for the mutant allele.

The comparison between the $\mathrm{AD}$ and $\mathrm{AR}$ models points up the basic limitation in discriminating between genetic models, which is often overlooked or treated too casually in studies of familial diseases; ie, that several common genetic models can adequately explain the phenotypes observed in a given pedigree. For this pedigree structure and these allele frequencies, it is possible for the $\mathrm{AD}$ model to be 5 times more likely than the AR model or for the AR model to be 13 times more likely than the AD, but no stronger statement of support is possible. Altering the pedigree structure or the allele frequency will change the limits on the "model choice" statistic $\Lambda$. For example, at these allele frequencies, if there were five offspring per pedigree, the lower limit on $\Lambda$ would be -1.42 ; for six offspring it would be-1.72. Since the upper limit results from identical expected phenotypic distributions among offspring, this limit would not be affected by increasing the sibship size. Altering the allele frequency can, however, change the upper limit on $\Lambda$ for the "affected" $X$ "unaffected" mating and even permit the "affected" X "affected," mating to be observed in small samples (which has no upper limit).

While the theoretical limits on the "model choice" statistic can be expressly defined for every possible combination of qualitative phenotypes (such as "affected" vs "unaffected") in a pedigree of this size, to date it has not been possible to derive analytically the distribution of $\Lambda$ when the phenotypes are quantitative. As seen in Figure $2 b$, however, if two genotypes have the equivalent of three standard deviations between their phenotypic means, most pedigrees of this size will give maximum permissible support to the correct autosomal model.

For comparison between X-linked models, of course, the sex of the proband parent and the sex distribution among offspring become important. Table I lists the theoretical limits on pairwise comparisons of four single locus, two allele models (autosomal dominant, $\mathrm{AD}$; autosomal recessive, $\mathrm{AR}$; X-linked dominant, $\mathrm{XD}$; X-linked recessive, $\mathrm{XR}$ ) separately for pedigrees with a male proband (the upper right triangular half of Table I) and with a female proband (the lower left triangular half of Table I). These limits apply to matings where the spouse of the proband is "unaffected." These limits were obtained by letting all possible combinations of discrete phenotypes serve as the extreme case of a quantitative phenotype. As illustrated in Figure 2, with poor phenotypic resolution the "model choice" statistic may be relatively far from its limit, but by improving phenotypic resolution it will rapidly approach the appropriate limit.

Table I requires some interpretation. The absence of a limit on the "model choice" statistic implies that one model predicts combinations of phenotypes which are impossible under the other model. For example, the AD model predicts both "unaffected" daughters and "affected" sons from a male proband (and an "unaffected" spouse). Under the XD mode1, on the other hand, the probability of either an "unaffected" daughter or an "affected" son is zero. The exact value of the "model choice" statistic will, of course, depend on the likelihood of the daughter's observed phenotype being drawn from the "unaffected" distribution and, similarly, that of a son's phenotype being drawn from the "affected" distribution. Nonetheless if a pedigree with a male proband is actually AD, a strong statement of support for that model (over the XD model) is possible. The converse is not true, however, for an XD pedigree with a male proband. The maximum level of support possible, given in Table $\mathrm{I}$, is a function of the allele frequencies involved. 
Another point to be realized about Table I is that sometimes these limits define an extremely narrow range, and when this occurs the pedigree can be considered non-informative for the comparison in question. For example, a pedigree with a female proband is noninformative for the $\mathrm{AD}$ vs $\mathrm{XD}$ comparison. Even for relatively common diseases $(1 \%-10 \%$ prevalence), these limits define a very narrow range around $\Lambda=0$, ie, the point where both models are equally likely. Where the disease has a prevalence of $1 \%$, the "model choice" statistic $\Lambda[=\log \mathrm{L}(\mathrm{AD}) / \mathrm{L}(\mathrm{XD})]$ can vary between 0.0025 and -0.0033 ; with $10 \%$ prevalence, $\Lambda$ can vary between 0.014 and- 0.035 . This tight range on $\Lambda$ results from the identical phenotypic distribution among children of fernale probands expected under these two models and the similar allele frequencies implied by the observed prevalence. Pedigrees of this structure with a male proband can also be non-informative, as shown in the comparison of the AR and XR models. Again, since the expected phenotypic distributions among offspring of a male proband are identical under these two models, the limits on the "model choice" statistic $\Lambda[=\log \mathrm{L}(\mathrm{AR}) / \mathrm{L}(\mathrm{XR})]$ define a narrow range around zero for relatively common diseases.

Sometimes the sex distribution among the offspring is the critical factor. For example, pedigrees with a male proband and no daughters cannot be used to discriminate between the two sex-linked models $\mathrm{XD}$ and $\mathrm{XR}$, because the hemizygous males provide no information about the dominance or recessivity of the trait. Similarly, pedigrees with a male proband and no daughters cannot be used to discriminate between the AR and XD models. Conversely, pedigrees of this structure with a female proband and no sons are noninformative for the AR and XR models.

\section{Choosing From Several Single Locus Models}

In some cases, the study design may be to choose among several competing models rather than the pairwise comparisons discussed above. For example, if a disease were thought to be heterogeneous in its genetic origins, any sample of pedigrees ascertained through the disease could contain pedigrees in which the disease was $\mathrm{AD}$, others in which it was AR, etc. A "model choice" analysis was applied to the pedigrees simulated during the course of this study for the four single locus models: $\mathrm{AD}, \mathrm{AR}, \mathrm{XD}$, and $\mathrm{XR}$. The likelihood of each model was computed on every pedigree and the best supported model was chosen. From these data, no minimum level of support was required, ie, the most likely model was chosen even if it was only slightly better than another model. Table II gives the actual results for these 640 simulated pedigrees using two levels of phenotypic resolution. While this sample size is too small for precise estimates of the probability of choosing correctly among these four models, Table II does clearly show that improving phenotypic resolution increases the probability of choosing the true model for informative pedigree structures. For example, only $54 \%$ of $\mathrm{AD}$ pedigrees with a male proband favored the AD model when phenotypic resolution was poor; however, $75 \%$ did so when phenotypic resolution was improved. When a pedigree is non-informative for a pairwise comparison (eg, pedigrees with a male proband are non-informative for the AR vs XR comparison), often it will favor the wrong model altogether. This explains the absence of pedigrees with a male proband supporting the AR model, even when they are truly AR. While the difference in the likelihoods of the AR and XR models on these non-informative pedigrees is small, it is not possible to identify certain models accurately. 
TABLE I. Theoretical Limits on the "Model Choice" Statistic $\Lambda$, for Matings Between an "Affected" Proband and an "Unaffected" Spouse With s Sons and $d$ Daughters $(s+d=n$, the total number of offspring)

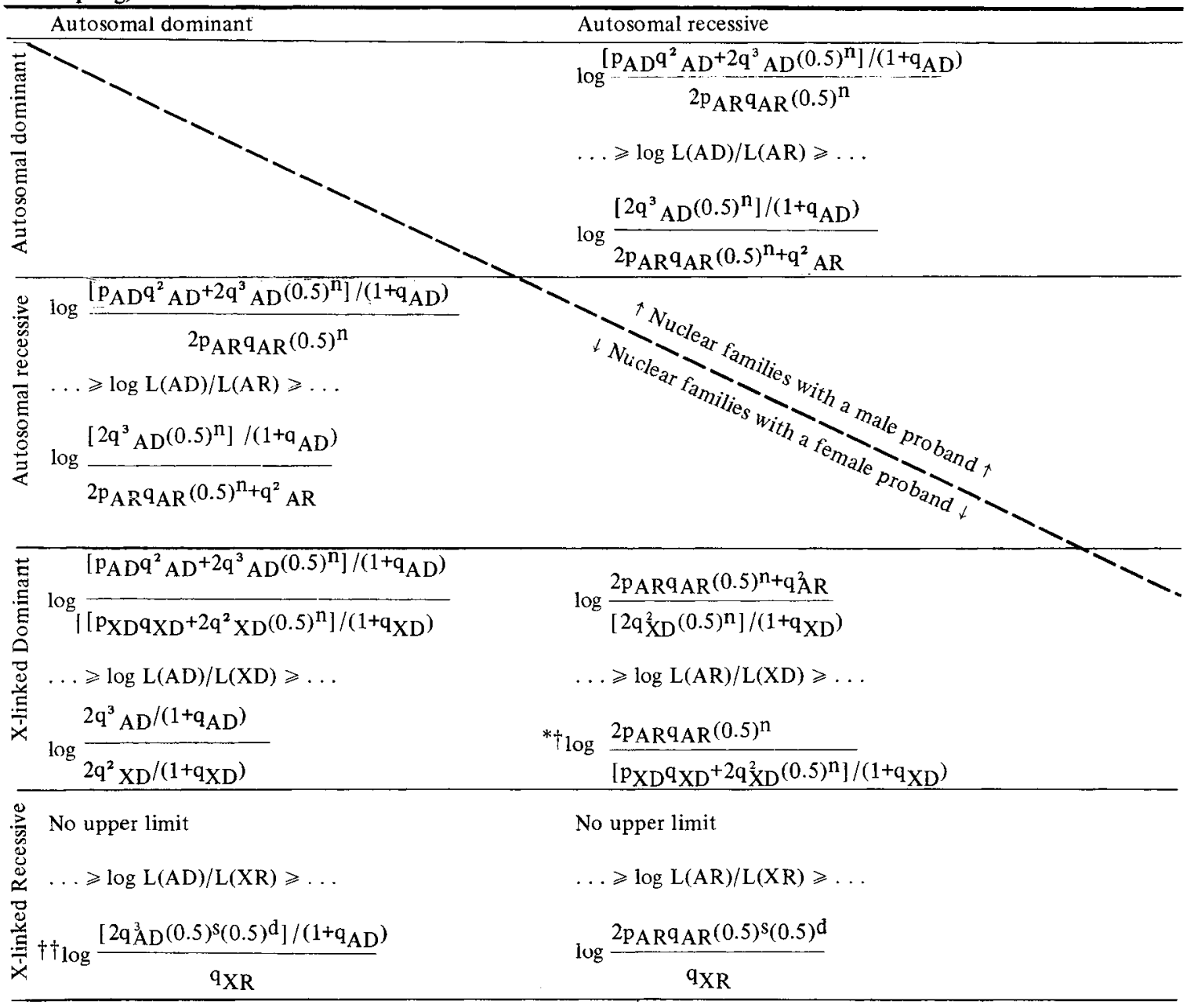

$*$ If there are no sons, the limit is $\frac{\left[p_{A D} q^{2} A D+2 q^{3} A D^{(0.5)}\right] /\left(1 .+q_{A D}\right)}{q X D}$

**If there are no daughters, the limit is $\log \frac{2 p_{A R} q_{A R}(0.5)^{s}+q_{A R}^{2}}{q^{2}}$

$\dagger$ If there are no daughters, the limit is $\log \frac{q_{X}^{2} D}{2 p_{X R}{ }^{q}{ }_{X R}^{(0.5)^{s}+q_{X R}^{2}}}$

$\dagger \dagger$ If there are no sons, the limit is $\log 2 \mathrm{p}_{\mathrm{AR}} \mathrm{q}_{\mathrm{AR}}{ }^{(0.5)^{\mathrm{d}_{+}} \mathrm{q}_{A R}^{2}}$

$$
\text { qXR }
$$




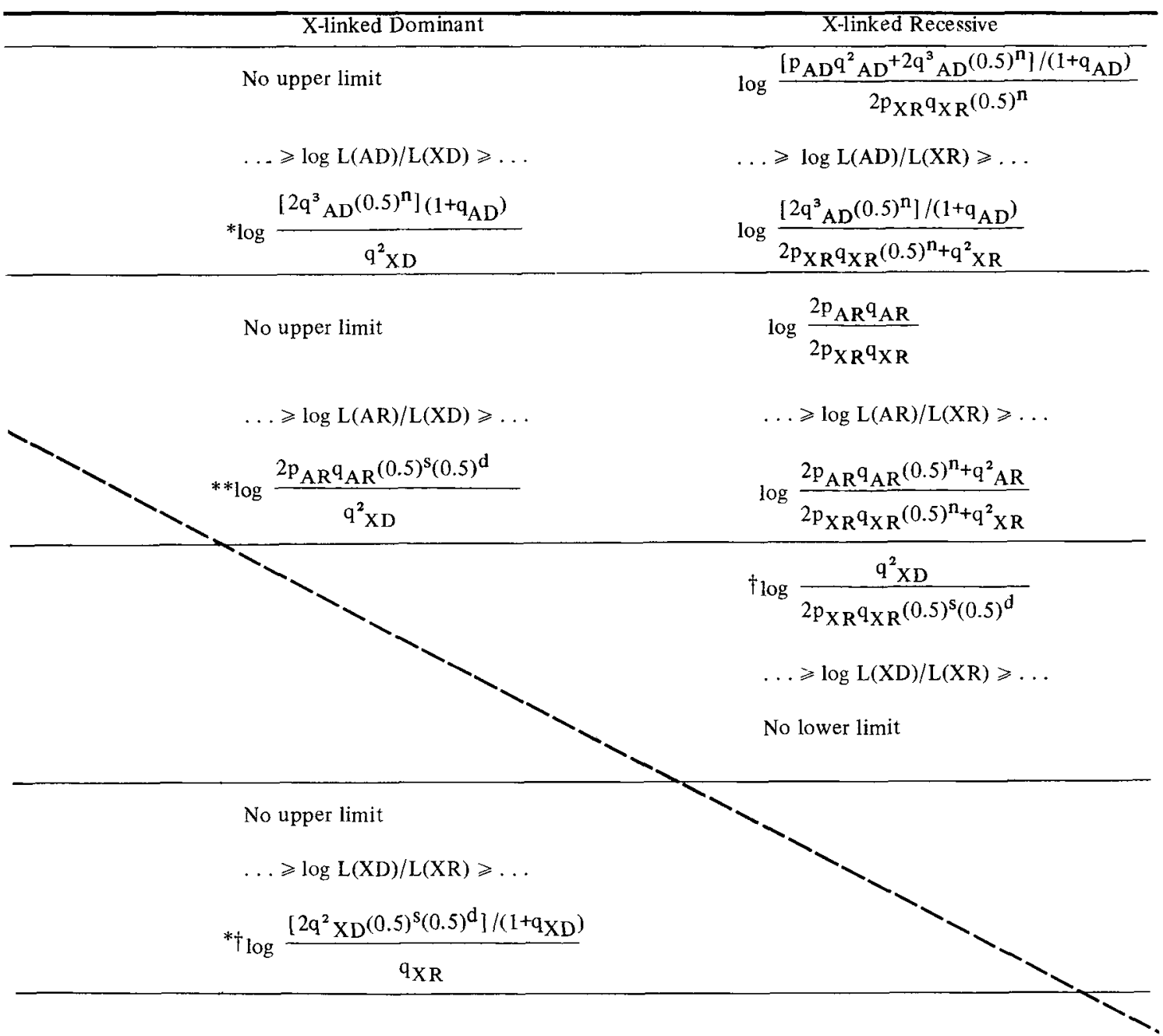

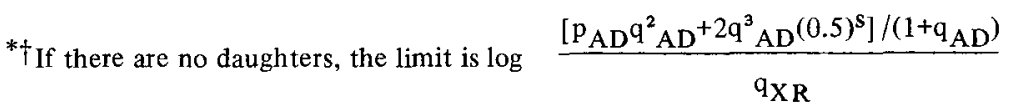

Similarly for the $\mathrm{XD}: \mathrm{XR}$ comparison.

The frequency of the mutant allele, $p$, is obtained by assuming Hardy-Weinberg equilibrium in the population given an observed prevalence of the disease. 
TABLE II. Choosing the Best-Supported Genetic Model Among the Four Single Locus Models Used to Simulate 640 Pedigrees

\begin{tabular}{|c|c|c|c|c|c|c|c|c|c|c|c|c|}
\hline \multirow{4}{*}{$\begin{array}{l}\text { Univariate } \\
\text { phenotypes }\end{array}$} & \multirow{2}{*}{\multicolumn{6}{|c|}{$\begin{array}{l}\text { Pedigrees with } \\
\text { a male proband } \\
\text { Chosen model }\end{array}$}} & \multirow{2}{*}{\multicolumn{6}{|c|}{$\begin{array}{l}\text { Pedigrees with } \\
\text { a female proband } \\
\text { Chosen model }\end{array}$}} \\
\hline & & & & & & & & & & & & \\
\hline & & $\mathrm{AD}$ & AR & $\mathrm{XD}$ & XR & Total & & $\mathrm{AD}$ & $\mathrm{AR}$ & $\mathrm{XD}$ & XR & Total \\
\hline & $\mathrm{AD}$ & $\begin{array}{c}43 \\
(54 \%)\end{array}$ & 0 & 20 & 17 & 80 & $\mathrm{AD}$ & $\begin{array}{l}10 \\
(13 \%)\end{array}$ & 19 & 32 & 19 & 80 \\
\hline True & $\mathrm{AR}$ & 13 & $\begin{array}{c}0 \\
(0 \%)\end{array}$ & 18 & 49 & 80 & AR & 3 & $\begin{array}{c}47 \\
(59 \%)\end{array}$ & 15 & 15 & 80 \\
\hline \multirow[t]{3}{*}{ Model } & $\mathrm{XD}$ & 4 & 0 & $\begin{array}{c}39 \\
(81 \%)\end{array}$ & 5 & 48 & $\mathrm{XD}$ & 10 & 22 & $\begin{array}{c}34 \\
(35 \%)\end{array}$ & 30 & 96 \\
\hline & $\mathrm{XR}$ & 20 & 0 & 23 & $\begin{array}{l}101 \\
(70 \%)\end{array}$ & 144 & $\mathrm{XR}$ & 1 & 6 & 1 & $\begin{array}{c}24 \\
(75 \%)\end{array}$ & 32 \\
\hline & Total & 80 & 0 & 100 & 172 & 352 & Total & 24 & 94 & 82 & 88 & 288 \\
\hline \multirow[t]{2}{*}{$\begin{array}{l}\text { Quadrivaria } \\
\text { phenotypes }\end{array}$} & & $\mathrm{AD}$ & AR & $\mathrm{XD}$ & XR & Total & & $\mathrm{AD}$ & AR & XD & XR & Total \\
\hline & $\mathrm{AD}$ & $\begin{array}{c}60 \\
(75 \%)\end{array}$ & 0 & 12 & 8 & 80 & $\mathrm{AD}$ & $\begin{array}{c}7 \\
(9 \%)\end{array}$ & 12 & 48 & 13 & 80 \\
\hline True & $\mathrm{AR}$ & 14 & $\begin{array}{c}1 \\
(1 \%)\end{array}$ & 10 & 55 & 80 & AR & 0 & $\begin{array}{c}58 \\
(73 \%)\end{array}$ & 14 & 8 & 80 \\
\hline \multirow[t]{3}{*}{ Model } & XD & 2 & 0 & $\begin{array}{c}45 \\
(94 \%)\end{array}$ & 1 & 48 & $\mathrm{XD}$ & 7 & 9 & $\begin{array}{c}68 \\
(71 \%)\end{array}$ & 12 & 96 \\
\hline & XR & 8 & 0 & 12 & $\begin{array}{l}124 \\
(86 \%)\end{array}$ & 144 & $\mathrm{XR}$ & 0 & 1 & 1 & $\begin{array}{c}30 \\
(94 \%)\end{array}$ & 32 \\
\hline & Total & 84 & 1 & 79 & 188 & 352 & Total & 14 & 80 & 131 & 63 & 288 \\
\hline
\end{tabular}

Abbrebiations: AD, autosomal dominant; $\mathrm{AR}$, autosomal recessive; $\mathrm{XD}, \mathrm{X}$-linked dominant; $\mathrm{XR}, \mathrm{X}$-linked recessive.

Aside from this problem, however, this "model choice" analysis has revealed the existing genetic heterogeneity in the total set of simulated pedigrees. The resulting groups of pedigrees supporting these four models are by no means homogeneous, but they could serve as a branching point in a study designed to identify and describe separate genetic forms of a familial disease.

\section{DISCUSSION}

This "model choice" approach was originally designed to assist in detecting genetic heterogeneity in familial diseases, rather than to identify a single genetic mechanism behind a homogeneous disease. By identifying subgroups of pedigrees which support one model over another and then focusing further analyses on these separate groups, it may be possible to distinguish between different genetic forms of a disease. Such subgroups of pedi- 
grees could be examined either by more extensive phenotypic evaluation or by further genetic analysis, eg, linkage studies or further analysis of an expanded pedigree identified in this manner. This study using simulated data on small pedigrees ( 2 parents, 4 offspring) shows the study design is feasible, especially if there is reasonably good resolution of phenotypes.

The results of this study, however, also point up some important and perhaps critical limitations which must be considered. First and foremost, the pedigree structure at hand and the limitations it imposes on discriminating between different single locus models must be carefully examined. Certain pedigrees are totally non-informative for comparisons between certain genetic models. Other pedigrees have fixed limits on the degree of support they can give one model or another. Such limits occur when both genetic models can explain the observed data at slightly different frequencies. Second, improving the phenotypic resolution by considering multivariate phenotypes can increase the probability of choosing the correct genetic model for a given pedigree up to, but not beyond, the theoretical limits just mentioned. As a consequence of these two points, careful consideration must be given to the design of genetic studies to avoid collecting non-informative pedigrees whenever possible. For example, if one were interested in discriminating between the $\mathrm{AD}$ and XD models, it would be unwise to sample nuclear families by ascertaining through an affected mother. Of course, it will not always be possible to known in advance if a pedigree is informative or not because the exact structure of the pedigree determines its potential for discriminating among models. In addition, consideration must be given to the selection of competing genetic models which will be considered. The "shotgun" approach of considering a wide field of genetic models should be avoided. As shown in this study, small pedigrees can have severe limitations on their ability to discriminate among four divergent single locus models. With larger pedigrees, the probability of severe constraints on the choice between genetic models will drop, but any given pedigree structure may or may not be informative for a particular comparison. As these simulated pedigrees show, however, even small pedigrees with definite limits on the ability to discriminate between models can be useful in detecting genetic heterogeneity, especially when multivariate phenotypes are incorporated into the analysis to improve phenotypic resolution.

\section{CONCLUSIONS}

This work on simulated pedigrees illustrates the difficulty in detecting genetic heterogeneity in a familial disease. While it is clearly possible to identify groups of pedigrees supporting different genetic models with reasonable overall confidence that such groups, to some degree, reflect underlying differences in genetic mechanisms, such an approach cannot overcome the limitations imposed by overlapping predictions of these different genetic models. These limitations result in some pedigrees being non-informative for certain comparisons. The non-informative nature of some pedigrees may seem obvious after considering the genetic models carefully, but such careful consideration is not always given during the design and sampling phases of genetic studies.

Equal consideration must also be given to the interpretation of the results of this type of analysis. For example, the failure of pedigrees with a male proband to support the AR model should not be interpreted as solid indication that there is no AR form of the disease until the pedigrees with a female proband (which do support the AR model) are considered. While these newly defined groups of pedigrees are ideal starting points for further data collection and subsequent reanalysis, it must be realized that they are not permanent. For example, an AR pedigree with a male proband (currently misclassified as 
XR) may be correctly reclassified if further information on the proband's parents is obtained. This new, larger pedigree may no longer be non-informative. The "model choice" approach described here should be viewed as one step of many which can be used to identify genetic heterogeneity in familial diseases.

\section{ACKNOWLEDGMENTS}

I would like to thank Drs. P. Smouse, C. Sing, and P. Moll for their comments and support throughout this project. I would also like to thank Drs. R. Elston, E. Thompson, S. Haastedt, T. Bishop, G. Chase, and C. Witten for their comments on versions of this work. Parts of this project were supported by grant NIH-I-Tol-GM-71.

\section{REFERENCES}

Beaty TH: Likelihood analysis of multivariate phenotypes in pedigrees. PhD dissertation, University of Michigan, Ann Arbor, 1978.

Cannings C, Thompson EA, Skolnick M: Probability functions on complex pedigrees. Adv Appl Prob 10:26-61, 1978.

Elston RC, Namboodiri KK, Glueck CJ, Fallat R, Tsang R, Leuba V: Study of the genetic transmission of hypercholesterolemia and hypertriglyceridemia in a 195 member kindred. Ann Hum Genet $67-87,1975$.

Elston RC, Stewart J: A general model for the genetic analysis of pedigree data. Hum Hered 21: 523-542, 1971.

Go RCP, Elston RC, Kaplan EB: Efficiency and robustness of pedigree segregation analysis. Am J Hum Genet 30:28-37, 1978.

Lange K, Elston RC: Extensions to pedigree analy sis. I. Likelihood calculations for simple and complex pedigrees. Hum Hered 25:95-105, 1975.

MacLean CJ, Morton NE, Lew R: Analysis of family resemblance. IV. Operational characteristics of segregation analy sis. Am J Hum Genet 27:365-384, 1975.

Edited by Edmond A. Murphy 